\title{
Resonant trans-Neptunian objects as a source of Jupiter-family comets
}

\author{
E. L. Kiseleva $\dagger$ and V. V. Emel'yanenko \\ Department of Computational and Celestial Mechanics, South Ural University, Russia \\ email: kleo@susu.ac.ru
}

\begin{abstract}
The dynamical interrelation between resonant trans-Neptunian objects and shortperiod comets is studied. Initial orbits of resonant objects are based on computations in the model of the outward transport of objects during Neptune's migration in the early history of the outer Solar system. The dynamical evolution of this population is investigated for 4.5 Gyr, using a symplectic integrator. Our calculations show that resonant trans-Neptunian objects give a substantial contribution to the planetary region. We have estimated that the relative fraction of objects captured per year from the $2 / 3$ resonance to Jupiter-family orbits with perihelion distances $q<2.5 \mathrm{AU}$ is $0.4 \times 10^{-10}$ near the present epoch.
\end{abstract}

Keywords. Trans-Neptunian objects, comets, resonances, migration.

\section{Introduction}

The dynamical and physical characteristics of short-period comets imply that these bodies originate from the outer Solar system. It was shown by Duncan \& Levison (1997) and by Emel'yanenko et al. (2004) that the scattered disc trans-Neptunian objects are a source of Jupiter-family comets. On the other hand, Morbidelli (1997), and Nesvorný \& Roig (2000) demonstrated that objects from the classical Edgeworth-Kuiper belt moving in the resonance $2 / 3$ with Neptune can also contribute to the population of Jupiter-family comets. The estimate of the transfer rate from the $2 / 3$ resonance to Jupiter-family comets is the main aim of the present paper.

We try to investigate the features of that resonant population which arises during the evolution under planetary perturbations for the age of the Solar system. The longterm motion in the $2 / 3$ resonance was studied by Morbidelli (1997). It was shown that the mechanism of the chaotic diffusion can drive Plutinos out of the $2 / 3$ resonance, after which they are subjected to close encounters with the giant planets and eventually evolve into Jupiter-family comets. However, Morbidelli (1997) studied a uniform distribution of initial orbits. Now we know that the mechanism of Neptune's migration is very important in the formation of the resonant population structure (Malhotra 1993, 1995; Hahn \& Malhotra 1999, 2005).

Therefore, we analyze the distribution of resonant objects which arises after the outward transport of objects during Neptune's migration in the early history of the Solar system and the evolution under planetary perturbations for the age of the Solar system. Our integrations are performed using the symplectic integrator by Emel'yanenko (2002). This explicit second-order integrator uses a time transformation which allows to adjust the time-step in correspondence with the distance from the Sun and the magnitude of perturbations. Thus it can handle both high-eccentricity orbits and close encounters with planets.

$\dagger$ Present address: South Ural University, 76 Lenina, Chelyabinsk, Russia, 454080 


\section{Model of migration}

We have tested the hypothesis that Neptune migrated outwards by several AU during the solar system's past and, in so doing, sculpted the pattern of resonance occupation in the Kuiper belt (Malhotra 1995).

The simulation parameters are identical to those in Malhotra (1995). They correspond to a smooth migration of Neptune. We consider four models with different values of initial inclinations and eccentricities. The eccentricities and inclinations of objects are distributed in the ranges $(0,0.001),(0,0.01),(0,0.05)$, and $(0,0.1)$, respectively, for the four models. Each set contains 1000 initial orbits in the range $(27,42)$ AU of semimajor axes.

We have found that dynamical features are similar in all models, and they are analogous to results of Malhotra (1995). Therefore, in the following we discuss only computations for the fourth model where the inclination distribution is the broadest, and is more consistent with the observed distribution of the $2 / 3$ resonant population than for the other models.

\section{Evolution for the age of the Solar system}

We have taken 332 objects located in the $2 / 3$ resonance region from the fourth scenario. The inclinations and eccentricities of these objects lie in the ranges $(0.9,22.1)$ degrees and $(0.01,0.33)$, respectively. These orbits have been integrated for $4.5 \mathrm{Gyr}$, unless objects reach orbits with perihelion distances $q<2.5$ au or evolve to hyperbolic orbits. We include perturbations from the four outer planets.

The number of particles surviving in the $2 / 3$ resonance after $4.5 \mathrm{Gyr}$ is 44 . Thus the $2 / 3$ resonance population after $4.5 \mathrm{Gyr}$ is approximately ten times as small as the original one. From 44 resonant objects, five objects were located in this region at the beginning of the planetary migration, and the others reached this region in the process of migration.

Figure 1 shows the initial and final distributions of semimajor axes and eccentricities for objects in the $2 / 3$ resonance.

\section{Evolution to Jupiter-family orbits}

Figure 2 shows the number $N$ of particles surviving in the resonance $2 / 3$ as a function of time for the last 2 Gyr of integrations. We have found that 7 objects reach the region $q<20 \mathrm{AU}$ for the last billion years of integrations. These objects are cloned 10 times. We study the orbital evolution of these objects until they are captured to $q<2.5$ au or evolve to a hyperbolic orbit. We then apply the method described in Emel'yanenko et al. (2004) to calculate the rate of injection of Jupiter-family comets from the $2 / 3$ resonant population.

We have estimated that the relative fraction of objects captured per year from the $2 / 3$ resonance to Jupiter-family orbits with perihelion distances $q<2.5 \mathrm{AU}$ is $0.4 \times 10^{-10}$ near the present epoch. This is approximately two times as large as the corresponding estimate in the model of Morbidelli (1997).

Figure 3 shows the distribution of Tisserand parameters and inclinations for Jupiterfamily comets with $q<2.5 \mathrm{AU}$, captured from the $2 / 3$ resonant population. This distribution is consistent with the observed distribution of Jupiter-family comets. However, this distribution is more concentrated towards high values of Tisserand parameter than that in models studying high-eccentricity trans-Neptunian objects (Emel'yanenko et al. 2004) and objects from the Oort cloud (Emel'yanenko et al. 2005) as sources of Jupiter-family comets. 

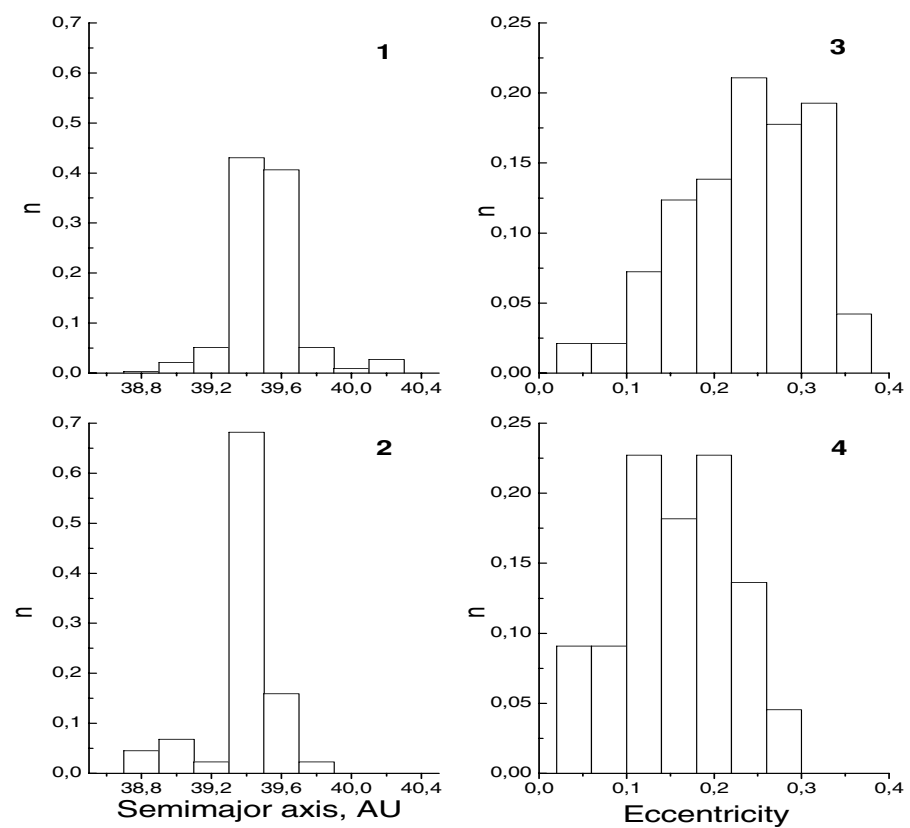

Figure 1. (1) The distribution of semimajor axes for objects in the $2 / 3$ resonance at the end of Neptune's migration. (2) The distribution of semimajor axes for objects in the $2 / 3$ resonance after 4.5 Gyr. (3) The distribution of eccentricities for objects in the $2 / 3$ resonance at the end of Neptune's migration. (4) The distribution of eccentricities for objects in the $2 / 3$ resonant objects after 4.5 Gyr.

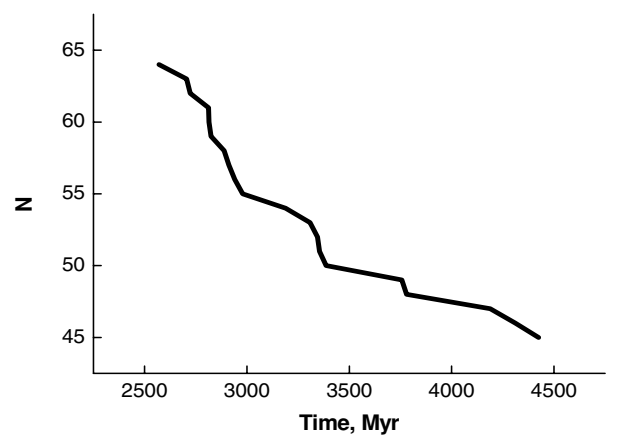

Figure 2. Evolution of the number $N$ of particles survivng in the resonance $2 / 3$ as a function of time.

\section{Conclusions}

We studied the dynamical connection between resonant trans-Neptunian objects and short-period comets in the model based on the origin of objects by the outward transport during Neptune's migration and the subsequent evolution under planetary perturbations for 4.5 Gyr. Our calculations show that the relative fraction of objects captured per year from the $2 / 3$ resonance to Jupiter-family orbits with perihelion distances $q<2.5$ AU is $0.4 \times 10^{-10}$ near the present epoch. This estimate is close to that obtained earlier for scattered disc objects as a source of Jupiter-family comets (Emel'yanenko et al. 2004). 


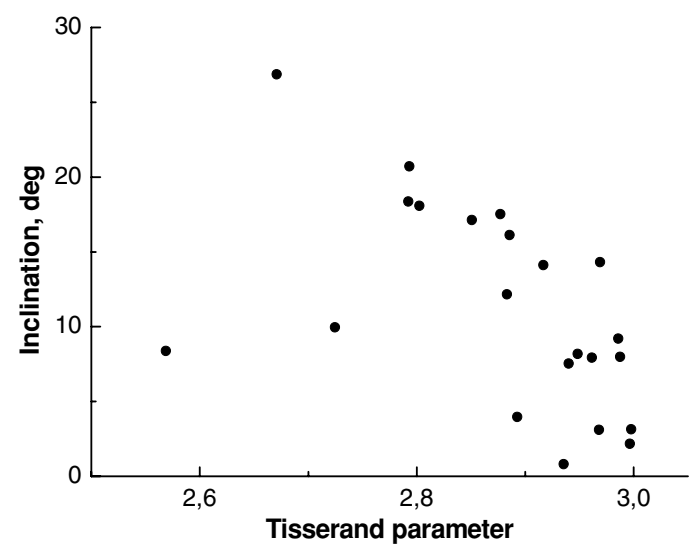

Figure 3. The distribution of Tisserand parameters and inclinations for simulated objects from the $2 / 3$ resonant population when their perihelia first drop below $2.5 \mathrm{AU}$.

Therefore, the relative number of Jupiter-family comets originating from the $2 / 3$ resonance and the scattered disc should be approximately the same as the ratio of these populations. Thus the contribution of resonant trans-Neptunian objects to the population of Jupiter-family comets is not negligible.

\section{Acknowledgments}

This work was supported by RFBR Grants 04-02-96042 and 06-02-16512. The authors are grateful to Giovanni Valsecchi for helpful suggestions concerning the style of this paper.

\section{References}

Duncan, M.J. \& Levison, H.F. 1997, Science 276, 1670

Emel'yanenko, V.V. 2002, Celest. Mech. EJ Dyn. Astron. 84, 331

Emel'yanenko, V.V., Asher, D.J. \& Bailey, M.E. 2004, Mon. Not. R. Astron. Soc. 350, 161

Emel'yanenko, V.V., Asher, D.J. \& Bailey, M.E. 2005, Mon. Not. R. Astron. Soc. 361, 1345

Hahn, J.M. \& Malhotra, R. 1999, AJ 117, 3041

Hahn, J.M. \& Malhotra, R. 2005, AJ 130, 2392

Malhotra, R. 1993, Nature 365, 819

Malhotra, R. 1995, AJ 110, 420

Morbidelli, A. 1997, Icarus 127, 1

Nesvorný, D. \& Roig, F. 2000, Icarus 148, 282 